\title{
Asymptotic solutions for scattered field by a coated conducting cylinder with a thin lossy dielectric material
}

\author{
Keiji Goto $^{\text {a) }}$ and Le Hoang Loc \\ Department of Communication Engineering, National Defense Academy, \\ 1-10-20 Hashirimizu, Yokosuka, 239-8686 Japan \\ a)keigoto@nda.ac.jp
}

Abstract: We derive the novel asymptotic solutions for the scattered field when a cylindrical wave is incident on a coated conducting cylinder with a thin lossy dielectric material. We show that the extended UTD (uniform geometrical theory of diffraction) solution and the modified UTD solution derived by retaining the second order term are uniformly applicable in the transition region near the shadow boundary and in the deep shadow region in which the conventional UTD shadow region solution produces the substantial errors. The validity of the asymptotic solutions proposed here is confirmed by comparing with the exact solution.

Keywords: asymptotic solution, scattered field, coated conducting cylinder, thin lossy dielectric material

Classification: Electromagnetic theory

\section{References}

[1] P. H. Pathak, "An asymptotic analysis of the scattering of plane waves by a smooth convex cylinder," Radio Sci., vol. 14, no. 3, pp. 419-435, May-June 1979.

[2] H. T. Kim and N. Wang, "UTD solution for electromagnetic scattering by a circular cylinder with thin lossy coatings," IEEE Trans. Antennas Propag., vol. 37, no. 11, pp. 1463-1472, Nov. 1989.

[3] T. B. A. Senior and J. L. Volakis, eds., Approximate Boundary Conditions in Electromagnetics, Chap. 7, IEE, London, 1995.

[4] J. B. Keller, "Diffraction by a convex cylinder," IRE Trans. Antennas Propag., vol. 4, pp. 312-321, July 1956.

[5] P. Hussar and R. Albus, "On the asymptotic frequency behavior of uniform GTD in the shadow region of a smooth convex surface," IEEE Trans. Antennas Propag., vol. 39, no. 12, pp. 1672-1680, Dec. 1991.

[6] T. Ida and T. Ishihara, "Novel high-frequency uniform asymptotic solution for scattered field by a conducting cylinder," IEICE Trans. Commun. (Japanese Edition), vol. J87-C, no. 10, pp. 754-767, Oct. 2004.

[7] T. Ida, T. Ishihara, and K. Goto, "Frequency-domain and time-domain novel uniform asymptotic solutions for scattered fields by an impedance cylinder and a dielectric cylinder," IEICE Trans. Electron., vol. E88-C, no. 11, pp. 2124-2135, Nov. 2005. 
[8] K. Goto, L. H. Loc, T. Kawano, and T. Ishihara, "Asymptotic analysis of high-frequency scattered fields by a coated conducting cylinder," The Papers of Technical Meeting on Electromagnetic Theory, IEE Japan, EMT-11-128, pp. 1-6, Nov. 2011 (in Japanese).

[9] M. Abramowitz and I. A. Stegun, eds., Handbook of Mathematicl Functions, pp. 358-478, Dover, New York, 1972.

[10] G. L. James, ed., Geometrical Theory of Diffraction for Electromagnetic Waves, 3rd ed., chap. 2, Peter Peregrinus Ltd., London, 1986.

\section{Introduction}

The studies in the high-frequency scattering by a smooth convex cylinder have been important research subjects for a variety of applications in the area of the radiation patterns of antennas mounted near curved surfaces such as an aircraft fuselage and the radar cross section of an airborne vehicle [1, 2, 3].

Pathak [1] considered the problem of scattering from a perfectly conducting cylinder and proposed the UTD (uniform GTD: geometrical theory of diffraction) solution which remains uniformly in the transition region near the shadow boundary (SB) and reduces automatically to the Keller's GTD solution [4] exterior to the transition region. The product of a wavenumber and a radius of curvature is sufficiently large, and when an observation point is moreover located to the deep shadow region comparatively near a cylinder, it has become clear that the UTD shadow region solution in [1] produces the substantial errors $[5,6,7]$.

Kim and Wang [2] proposed the UTD solution for the scattering from a circular cylinder with a thin lossy dielectric coating. However, it seems that the UTD shadow region solution in [2] becomes increasingly inaccurate with the increasing frequency because it has been derived by the same procedure given in [1].

In this paper, we derive an extended UTD solution and a modified UTD solution for the scattered field applicable uniformly in the transition region near the SB and in the deep shadow region when a cylindrical wave is incident on a coated conducting cylinder with a thin lossy dielectric material [8]. We will confirm the validity of the asymptotic solutions derived here by comparing with the exact solution calculated from the eigenfunction expansion $[2,3,8]$.

\section{Novel asymptotic solutions for the scattered field}

\subsection{Integral representation for the scattered field}

Fig. 1 (a) shows a coated conducting cylinder of radius $a$ with a thin lossy dielectric material of thickness $t(=a-b)$ and coordinate systems $(x, y, z)$ and $(\rho, \phi)$. We assume that the coated conducting cylinder and the electric line source are placed in parallel and are extended from $-\infty$ to $+\infty$ in the $z$-direction. 


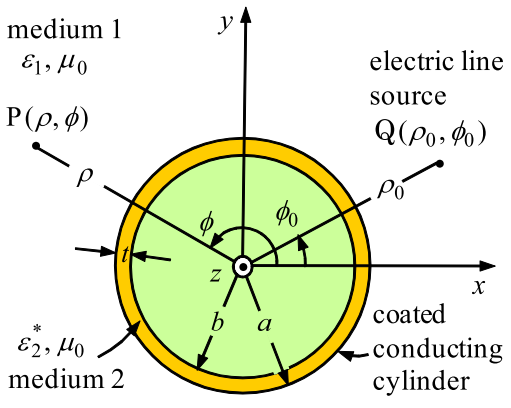

(a)

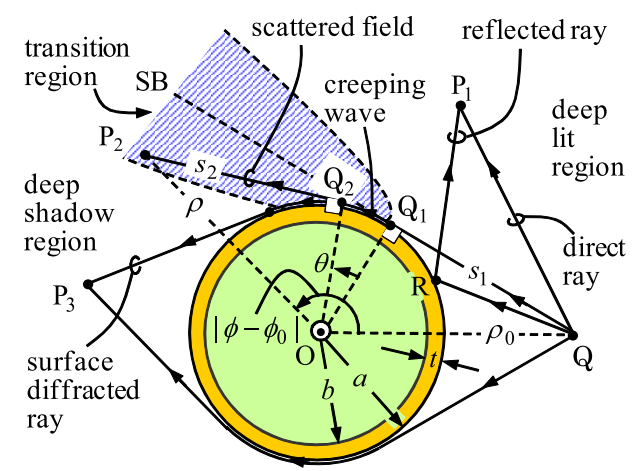

(b)

Fig. 1. Scattering phenomena by a coated conducting cylinder. (a) Coated conducting cylinder and coordinate systems $(x, y, z)$ and $(\rho, \phi)$. Schematic figure for the scattering observed at the points $\mathrm{P}_{1}, \mathrm{P}_{2}$, and $\mathrm{P}_{3}$.

The integral representation for the scattered electric field $E_{z}^{d}(\mathrm{P})$ arriving at the observation point $\mathrm{P}(\rho, \phi)$ from the counterclockwise direction without encircling the coated conducting cylinder after radiated from the electric line source $\mathrm{Q}\left(\rho_{0}, \phi_{0}\right)$ may be given as follows [8].

$$
\begin{gathered}
E_{z}^{d}(\mathrm{P})=-\frac{i}{8} \int_{-\infty}^{\infty} \frac{H_{\nu}^{(2)^{\prime}}\left(k_{1} a\right)+i Y_{\nu} H_{\nu}^{(2)}\left(k_{1} a\right)}{H_{\nu}^{(1)^{\prime}}\left(k_{1} a\right)+i Y_{\nu} H_{\nu}^{(1)}\left(k_{1} a\right)} H_{\nu}^{(1)}\left(k_{1} \rho_{0}\right) H_{\nu}^{(1)}\left(k_{1} \rho\right) e^{i \nu\left|\phi-\phi_{0}\right|} d \nu \\
Y_{\nu}=i \frac{Z_{1}}{Z_{2}} \cdot \frac{J_{\nu}^{\prime}\left(k_{2}^{*} a\right) Y_{\nu}\left(k_{2}^{*} b\right)-J_{\nu}\left(k_{2}^{*} b\right) Y_{\nu}^{\prime}\left(k_{2}^{*} a\right)}{J_{\nu}\left(k_{2}^{*} a\right) Y_{\nu}\left(k_{2}^{*} b\right)-J_{\nu}\left(k_{2}^{*} b\right) Y_{\nu}\left(k_{2}^{*} a\right)}, \quad Z_{1}=\sqrt{\frac{\mu_{0}}{\varepsilon_{1}}}, \quad Z_{2}=\sqrt{\frac{\mu_{0}}{\varepsilon_{2}^{*}}}
\end{gathered}
$$

Where, $H_{\nu}^{(1)}(\cdot)\left(H_{\nu}^{(2)}(\cdot)\right), J_{\nu}(\cdot)$, and $Y_{\nu}(\cdot)$ are respectively the Hankel function of the first (second) kind, the Bessel function, and the Neumann function [9] and the prime $\left({ }^{\prime}\right)$ on these functions denotes the derivative with respect to the argument. $k_{1}\left(=\omega \sqrt{\varepsilon_{1} \mu_{0}}\right)\left(k_{2}^{*}\left(=\omega \sqrt{\varepsilon_{2}^{*} \mu_{0}}\right)\right)$ and $Z_{1}\left(Z_{2}\right)$ are the wavenumber and the characteristic impedance in the surrounded medium 1 (in the dielectric medium 2). Notation $\varepsilon_{2}^{*}$ denotes the complex dielectric constant of the coating material and is given by $\varepsilon_{2}^{*}=\varepsilon_{2}+i \sigma / \omega$ where $\sigma$ is the conductivity. In (1), the time factor $\exp (-i \omega t)$ is suppressed in this paper.

The normalized admittance $Y_{\nu}$ in $(2)$ turns indeterminate when the imaginary part of the variables $k_{2}^{*} a$ and $k_{2}^{*} b$ is large. In order to solve the above-mentioned difficulty of $Y_{\nu}$ in (2), the approximate expression for $Y_{\nu}$ obtained by using the approximations for the cylindrical functions in the Appendix A under the condition that $\left|k_{2}^{*} a\right|$ and $\left|k_{2}^{*} b\right|$ become greater than $|\nu|$ $\left(\left|k_{2}^{*} a\right|,\left|k_{2}^{*} b\right|>|\nu|\right)$ is used and is given as follows.

$$
\begin{gathered}
Y_{\nu} \sim i \frac{Z_{1}}{Z_{2}} \cdot \frac{\sqrt{\left(k_{2}^{*} a\right)^{2}-\nu^{2}}}{k_{2}^{*} a} \cot \left(B_{1, \nu}-B_{2, \nu}\right), \quad \text { for } \quad\left|k_{2}^{*} a\right|,\left|k_{2}^{*} b\right|>|\nu| \\
B_{1, \nu}=\sqrt{\left(k_{2}^{*} a\right)^{2}-\nu^{2}}-\nu \cos ^{-1}\left(\frac{\nu}{k_{2}^{*} a}\right), \quad B_{2, \nu}=\sqrt{\left(k_{2}^{*} b\right)^{2}-\nu^{2}}-\nu \cos ^{-1}\left(\frac{\nu}{k_{2}^{*} b}\right) .
\end{gathered}
$$


The validity of the approximation expression in (3) is clarified numerically in $[8]$.

\subsection{Extended UTD solution for the scattered field}

When the observation point is located in the transition region shown in Fig. 1 (b), the main contribution to the integral in (1) arises from the portion of the integration path near $\nu=k_{1} a$ in the complex $\nu$-plane. Therefore, one may substitute the Airy function approximations [6, 7] for the Hankel functions $H_{\nu}^{(1),(2)}\left(k_{1} a\right)$ and $H_{\nu}^{(1),(2)^{\prime}}\left(k_{1} a\right)$. The variable is changed from $\nu$ to $\tau$ via $\nu=k_{1} a+M \tau, M=\left(k_{1} a / 2\right)^{1 / 3}$. While, the Hankel functions $H_{\nu}^{(1)}\left(k_{1} \rho_{0}\right)$ and $H_{\nu}^{(1)}\left(k_{1} \rho\right)$ are replaced by their second order Debye's approximations [6, 7].

By performing the straightforward manipulation, one may obtain the following extended UTD solution [8] (see Fig. 1(b)):

$$
\begin{gathered}
E_{z}^{d}(\mathrm{P}) \sim E_{z, \text { in }}\left(\mathrm{Q}_{1}\right) T\left(\mathrm{Q}_{1}, \mathrm{Q}_{2}\right)\left[\exp \left(i k_{1} s_{2}\right) / \sqrt{s_{2}}\right] \\
T\left(\mathrm{Q}_{1}, \mathrm{Q}_{2}\right)=-M \sqrt{\frac{2}{k_{1}}} \exp \left(i k_{1} \ell\right)\left[-\frac{F(X)}{2 \xi \sqrt{\pi}} e^{-i \pi / 4}+p_{s}^{e}\left(\xi, L, q_{c}(\tau)\right)\right] .
\end{gathered}
$$

Here $E_{z \text {,in }}\left(\mathrm{Q}_{1}\right)$ denotes the incident cylindrical wave propagating the distance from the source $\mathrm{Q}$ to the diffraction point $\mathrm{Q}_{1}$ and $T\left(\mathrm{Q}_{1}, \mathrm{Q}_{2}\right)$ denotes the transmission function, which expresses the scattering phenomena occurring along the arc of the coating surface from the point $\mathrm{Q}_{1}$ to the other diffraction point $\mathrm{Q}_{2}$. The propagation distance $\ell$ of the creeping wave, the Fresnel function $F(X)$, and the extended Pekeris function $p_{s}^{e}\left(\xi, L, q_{c}(\tau)\right)$ are defined as follows.

$$
\begin{gathered}
\ell=a \theta, \quad \theta=\left|\phi-\phi_{0}\right|-\cos ^{-1}\left(a / \rho_{0}\right)-\cos ^{-1}(a / \rho) \\
F(X)=-i 2 X \exp \left(-i X^{2}\right) \int_{X}^{\infty} \exp \left(i \tau^{2}\right) d \tau, \quad X=\theta \sqrt{2 k_{1} L} / 2 \\
p_{s}^{e}\left(\xi, L, q_{c}(\tau)\right)=\frac{\mathrm{e}^{-i \pi / 4}}{2 \sqrt{\pi}} \int_{i \infty}^{0} \frac{w_{2}^{\prime}(\tau)-q_{c}(\tau) w_{2}(\tau)}{w_{1}^{\prime}(\tau)-q_{c}(\tau) w_{1}(\tau)} \exp \left[i \xi \tau+i \frac{M^{2}}{2 k_{1} L} \tau^{2}\right] d \tau \\
+\frac{\mathrm{e}^{-i \pi / 4}}{\sqrt{\pi}} \int_{0}^{\infty} \frac{A i^{\prime}(\tau)-q_{c}(\tau) A i(\tau)}{w_{1}^{\prime}(\tau)-q_{c}(\tau) w_{1}(\tau)} \exp \left[i \xi \tau+i \frac{M^{2}}{2 k_{1} L} \tau^{2}\right] d \tau \\
w_{1}(\tau)=A i(\tau)-i B i(\tau), \quad w_{2}(\tau)=A i(\tau)+i B i(\tau) \\
q_{c}(\tau)=i M Y_{\nu}(\tau), \xi=M \theta, L=\frac{s_{1} s_{2}}{s_{1}+s_{2}}, s_{1}=\sqrt{\rho_{0}^{2}-a^{2}}, s_{2}=\sqrt{\rho^{2}-a^{2}} .
\end{gathered}
$$

In (5), the term in the square brackets represents the cylindrical wave propagating the distance from the point $\mathrm{Q}_{2}$ to the observation point $\mathrm{P}_{2} . Y_{\nu}(\tau)$ in (11) denotes a function obtained by $\nu=k_{1} a+M \tau, M=\left(k_{1} a / 2\right)^{1 / 3}$, replacing $\nu$ by $\tau$ in (3).

In the derivation of the conventional UTD solution in [2], the $\tau^{2}$ terms in (9) are dropped by assuming $k_{1} L \rightarrow \infty$. This is the reason why the conventional UTD shadow region solution (see (47) in [2]) produces the substantial errors. While, the novel asymptotic solution in (5) coincides with the extended UTD solution for the scattering from the impedance cylinder in [7] 
when $Y_{\nu}$ in (2) is replaced by $i Z_{1} / Z_{2}$.

\subsection{Modified UTD solution for the scattered field}

When the observation point is located in the shadow region where $\xi(=M \theta)$ satisfies $\xi>0$, the integral in (9) is evaluated rigorously by applying the residue theorem. Then substituting the residue series solution into (6) and then (6) into (5) and performing the straightforward manipulation yield the following modified UTD solution [8] (see Fig. 1(b)):

$$
\begin{gathered}
E_{z}^{d}(\mathrm{P}) \sim E_{z, \text { in }}\left(\mathrm{Q}_{1}\right) \sum_{m=1}^{\infty}\left[D_{m}\left(\mathrm{Q}_{1}\right) A_{m}\left(\mathrm{Q}_{1}\right) \mathrm{e}^{i k_{1} \ell-\Omega_{m} \ell} D_{m}\left(\mathrm{Q}_{2}\right) A_{m}\left(\mathrm{Q}_{2}\right)\right] \frac{\exp \left(i k_{1} s_{2}\right)}{\sqrt{s_{2}}} \\
D_{m}\left(\mathrm{Q}_{1,2}\right)=\frac{\sqrt{M} \exp (i \pi / 24)}{\sqrt[4]{2 \pi k_{1}} A i^{\prime}\left(-\sigma_{m}\right) \sqrt{g_{m}}}, g_{m}=1-\frac{\tau_{m}}{\left[q_{c}\left(\tau_{m}\right)\right]^{2}}, q_{c}\left(\tau_{m}\right)=i M Y_{\nu_{m}}\left(\tau_{m}\right) \\
A_{m}\left(\mathrm{Q}_{1,2}\right)=\exp \left[-\frac{M^{2} \sigma_{m}^{2}}{2 k_{1} s_{1,2}} \exp (i \pi / 6)\right], \quad \Omega_{m}=\frac{M}{a} \sigma_{m} \exp (-i \pi / 6) .
\end{gathered}
$$

Note that the coefficients $A_{m}\left(\mathrm{Q}_{1}\right)$ and $A_{m}\left(\mathrm{Q}_{2}\right)$ in (12) are obtained from the $\tau^{2}$ terms in (9). The eigenvalue $\tau_{m}$ and the corresponding $\sigma_{m}$ and $\nu_{m}$ are obtained from the following characteristic equation

$$
\begin{gathered}
w_{1}^{\prime}\left(\tau_{m}\right)-q_{c}\left(\tau_{m}\right) w_{1}\left(\tau_{m}\right)=0, \quad m=1,2,3, \cdots \\
\tau_{m}=\sigma_{m} \exp (i \pi / 3), \quad \nu_{m}=k_{1} a+M \tau_{m}, \quad M=\left(k_{1} a / 2\right)^{1 / 3}
\end{gathered}
$$

and $Y_{\nu_{m}}\left(\tau_{m}\right)$ in (13) is defined by (3) with the replacement of $\nu$ by $\nu_{m}$ in (16).

The residue series in (12) converges when the observation point is located in the region satisfying the condition $\theta>-a \operatorname{Re}\left[\sigma_{m}\right] /\left(4 M^{2} L\right)[6,7] . \operatorname{Re}\left[\sigma_{m}\right]$ denotes the real part of $\sigma_{m}$. Hence the modified UTD solution in (12) can be applied even in the lit side in the transition region satisfying the above inequality.

The novel asymptotic solution in (12) is quite identical to the modified UTD solution for the impedance cylinder in [7] when $Y_{\nu_{m}}\left(\tau_{m}\right)$ in (13) is replaced by $i Z_{1} / Z_{2}$.

\section{Numerical results and discussions}

In this section, we perform the numerical calculations required to assess the validity of the novel asymptotic solutions derived in Sect. 2 .

In Fig. 2 (a), the scattered electric field (E-mode type) magnitude curves are calculated by using the asymptotic solutions for the scattering from a coated conducting cylinder with a thin lossy dielectric material. The numerical results correspond to the case when the source $\mathrm{Q}\left(\rho_{0}, \phi_{0}\right)$ and the observation point $\mathrm{P}(\rho, \phi)$ are located relatively close to the coating surface. Numerical parameters used in the calculations are given in the caption of 
Fig. 2. In this case, the SB (see Fig. $1(\mathrm{~b})$ ) is located at $\left|\phi-\phi_{0}\right|=78.0^{\circ}$ and the transition region is defined as a case where the variable of $X$ in (8) satisfies $-\sqrt{3.0} \leq X \leq \sqrt{3.0}$. The switching point $A$ from the geometrical optics (GO) solution to the extended UTD and the modified UTD solution is $\left|\phi-\phi_{0}\right|=55.5^{\circ}$.

The exact solution (— : solid curve) has been calculated from the eigenfunction expansion $[2,3,8]$. In the deep lit region $0.0^{\circ} \leq\left|\phi-\phi_{0}\right| \leq 55.5^{\circ}$ exterior to the transition region, the GO solution ( $\circ \circ \circ$ ) consisting of the direct and the reflected ray (see Fig. 1 (b)) is used in the calculation. While, in the region $55.5^{\circ} \leq\left|\phi-\phi_{0}\right| \leq 180.0^{\circ}$, which includes the deep shadow region far away from the SB, the extended UTD solution (- - : red dashed curve) in (5) and the modified UTD solution ( $\cdots \cdots \cdot$ : dotted curve) in (12) are applied and are calculated from the superposition of the scattered field in the counterclockwise direction and the one in the clockwise direction (see in Fig. $1(b)$ ). It is clarified that the GO solution ( $\circ \circ \circ)$, the extended UTD solution (- - ), and the modified UTD solution (.......) agree excellently with the exact solution (-) in the respective regions. In fact, the dotted line $(\cdots \cdots .$.$) can not be seen in the region 55.5^{\circ} \leq\left|\phi-\phi_{0}\right| \leq 180.0^{\circ}$ including the interference phenomena near $180.0^{\circ}$ since the dotted line (the modified UTD solution) coincides with the solid line (the exact solution) and the red dashed line (the extended UTD solution) (The same can be said of Fig. 2 (b), Fig. 3, and Fig. 4). Also shown in Fig. 2 (a) is the conventional UTD shadow region solution (see (47) in [2]) (the conventional UTD shadow region solution (- becomes increasingly inaccurate as the observation point $\mathrm{P}$ moves toward the deep shadow region.

In Fig. 2 (b), in order to examine the accuracy of the extended UTD solu-

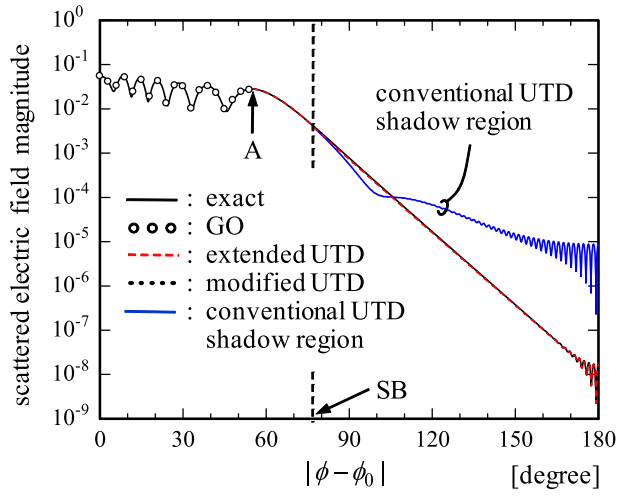

(a)

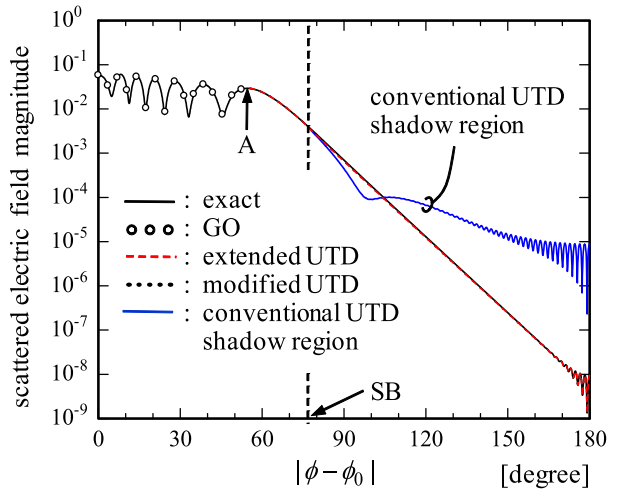

(b)

Fig. 2. Scattered electric field (E-mode type) magnitude vs. $\left|\phi-\phi_{0}\right|$ curves. The numerical parameters: $k_{1} a=100, a=5.0 \mathrm{~m}, t=0.2 \lambda, \varepsilon_{1}=\varepsilon_{0}\left(\varepsilon_{0}\right.$ : dielectric constant in the vacuum), source point: $\mathrm{Q}\left(\rho_{0}, \phi_{0}\right)=\left(1.4 a, 0.0^{\circ}\right)$, and observation point: $\mathrm{P}(\rho, \phi)=(1.2 a, \phi) . \quad(\mathrm{a})$ : dielectric material 2, $\varepsilon_{2}^{*}=\varepsilon_{2}+i \sigma / \omega\left(\varepsilon_{2}=5 \varepsilon_{0}, \sigma=0.053 \mathrm{~S} / \mathrm{m}\right),(\mathrm{b}): \mathrm{di}-$ electric material $2, \varepsilon_{2}^{*}=\varepsilon_{2}\left(\varepsilon_{2}=5 \varepsilon_{0}, \sigma=0.0 \mathrm{~S} / \mathrm{m}\right)$. 


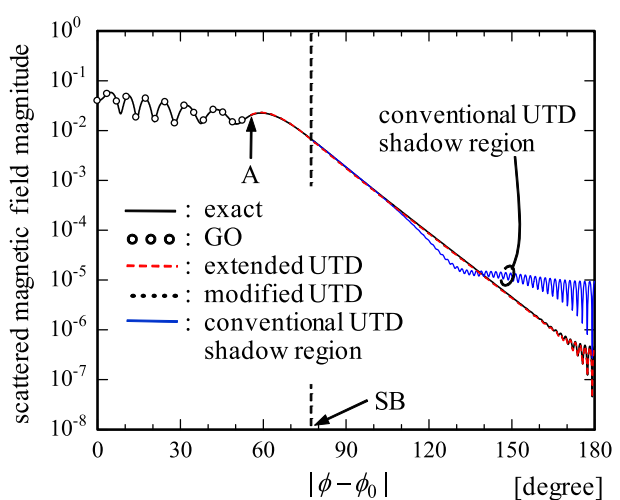

(a)

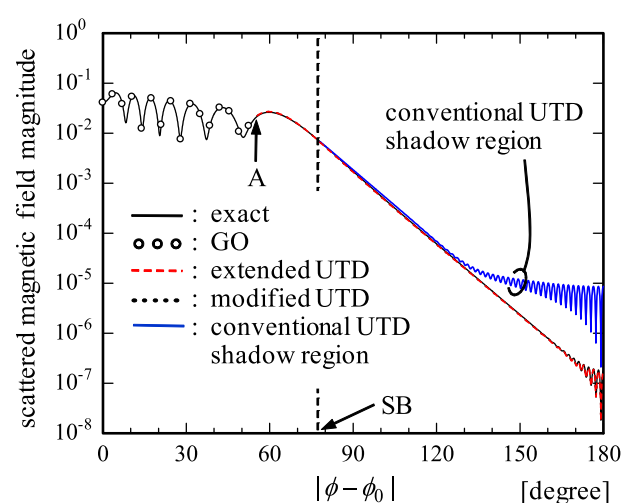

(b)

Fig. 3. Scattered magnetic field (H-mode type) magnitude vs. $\left|\phi-\phi_{0}\right|$ curves. (a): thin lossy dielectric material case, (b): thin lossless dielectric material case. The numerical parameters used in the calculation in Figs. 3 (a) and 3(b) are the same as those used in Figs. 2 (a) and 2 (b), respectively.

tion and the modified UTD solution for the low-loss property of the dielectric medium 2, we have shown the scattered electric field (E-mode type) magnitude curves by a coated conducting cylinder with a thin lossless dielectric material where the conductivity $\sigma=0$. It is observed that the GO solution ( $\circ \circ \circ$ ), the extended UTD solution (-- $)$ in (5), and the modified UTD solution $(\cdots \cdots)$ in $(12)$ agree excellently with the exact solution ( - ) in the respective regions. The accuracy of the extended UTD solution and the modified UTD solution by a coated conducting cylinder with a thin lossless dielectric material is assessed by the numerical results shown in Fig. 2 (b). While, one observed that the conventional UTD shadow region solution [2] (—) deviates increasingly as the observation point $\mathrm{P}$ moves toward the increasing $\left|\phi-\phi_{0}\right|$ direction in the shadow region.

We have shown in Figs. $3(\mathrm{a})$ and $3(\mathrm{~b})$ the scattered magnetic field (Hmode type) magnitude curves excited by a magnetic line source. Fig. 3 (a) corresponds to the scattering from the coated conducting cylinder with a thin lossy dielectric material. While, Fig. 3 (b) coincides with the scattering from the coated conducting cylinder with a thin lossless dielectric material where $\sigma=0$. The numerical parameters in Figs. 3 (a) and 3 (b) are the same as those used in Figs. 2 (a) and 2 (b), respectively. The results for the $\mathrm{H}$ mode type shown in Figs. 3 (a) and 3 (b) are similar to those for the E-mode type (Figs. 2 (a) and $2(\mathrm{~b})$ ), thereby the accuracy of the extended UTD and the modified UTD solution for the scattered magnetic field (H-mode type) is confirmed.

In Fig. 4 (a) and Fig. 4(b), in order to investigate the accuracy of the novel asymptotic solutions derived in Sect. 2 for the variation of the thickness $t(=a-b)$ of coating, we have shown the scattered electric field (E-mode type) magnitude curves when the thickness $t$ of the lossy dielectric material and that of the lossless dielectric material are changed to $t=0.1 \lambda$, 


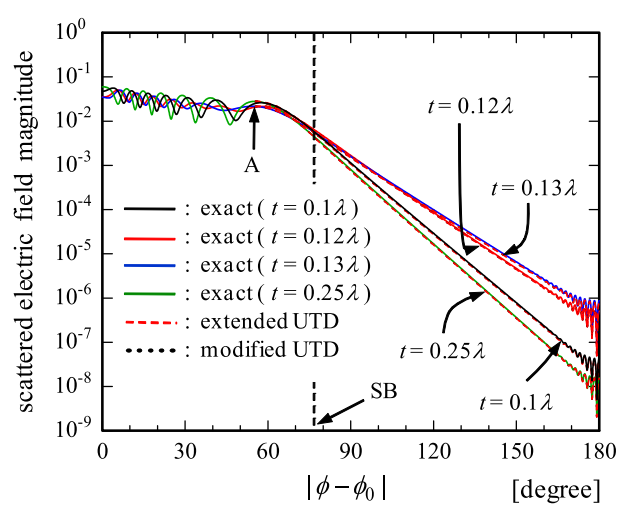

(a)

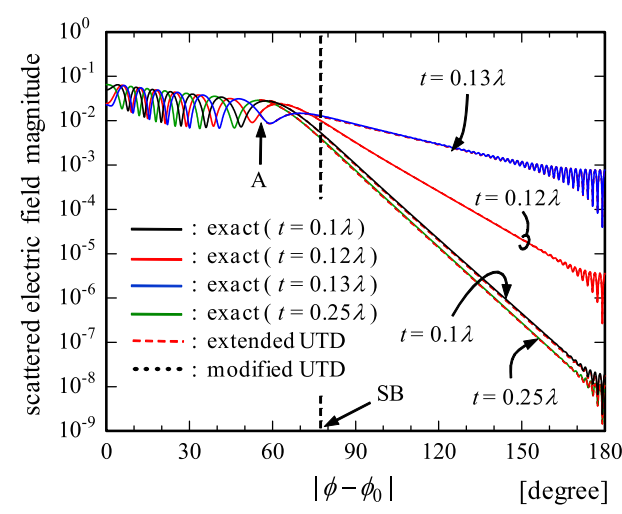

(b)

Fig. 4. Comparisons of the asymptotic solutions with the exact solution for the variation of the thickness $t$ of coating (E-mode type). The numerical parameters: $k_{1} a=100, a=5.0 \mathrm{~m}, \varepsilon_{1}=\varepsilon_{0}$, source point: $\mathrm{Q}\left(\rho_{0}, \phi_{0}\right)=\left(1.4 a, 0.0^{\circ}\right)$, and observation point: $\mathrm{P}(\rho, \phi)=(1.2 a, \phi)$. (a): dielectric material $2, \varepsilon_{2}^{*}=\varepsilon_{2}+i \sigma / \omega\left(\varepsilon_{2}=5 \varepsilon_{0}, \sigma=0.053 \mathrm{~S} / \mathrm{m}\right),(\mathrm{b}): \mathrm{di}-$ electric material 2, $\varepsilon_{2}^{*}=\varepsilon_{2}\left(\varepsilon_{2}=5 \varepsilon_{0}, \sigma=0.0 \mathrm{~S} / \mathrm{m}\right)$.

$0.12 \lambda, 0.13 \lambda$, and $0.25 \lambda$, respectively. The scattered electric fields observed at the observation point $\mathrm{P}$ located in the transition and the shadow region $55.5^{\circ} \leq\left|\phi-\phi_{0}\right| \leq 180.0^{\circ}$ may be obtained from the extended UTD solution in (5) or the modified UTD solution in (12). From the numerical results in Fig. 4 (a) and Fig. 4 (b), one may recognize that the extended UTD solution (-- ) and the modified UTD solution (.......) agree excellently with the exact solution (solid curve) to each thickness $t$. Each GO solution for the variation of the thickness $t$ of coating in the deep lit region $0.0^{\circ} \leq\left|\phi-\phi_{0}\right| \leq 55.5^{\circ}$ agrees excellently with the exact solution. However, in order to avoid the confusion in a figure, the numerical results of the GO solution are omitted in Fig. 4 (a) and Fig. 4 (b).

The validity of the extended UTD solution in (5) and the modified UTD solution in (12) for the scattered field by a coated conducting cylinder with a thin lossy dielectric material including the conductivity $\sigma=0$ is confirmed by the numerical results shown in Figs. 2, 3, and 4 .

\section{Conclusion}

We have derived the extended UTD solution and the modified UTD solution for the scattering from a coated conducting cylinder with a thin lossy dielectric material. The validity of the novel asymptotic solutions has been confirmed by comparing with the exact solution. We have clarified that the asymptotic solutions proposed here can be applied even in the deep shadow region where the conventional UTD shadow region solution produces the substantial errors. 


\section{Acknowledgement}

This work was supported in part by the Grant-in-Aid for Scientific Research

(C) (24560492) from Japan Society for the Promotion of Science (JSPS).

\section{A. Approximations for the cylindrical functions}

The approximations for the cylindrical functions applied in the text under the condition that $\left|k_{2}^{*} a\right|$ and $\left|k_{2}^{*} b\right|$ become greater than $|\nu|\left(\left|k_{2}^{*} a\right|,\left|k_{2}^{*} b\right|>|\nu|\right)$ are listed in this appendix (see (62) and (68) in Chap. 2 in [10]).

$$
\begin{aligned}
& J_{\nu}\left(k_{2}^{*} a\right) \sim \sqrt{\frac{2}{\pi}} \frac{1}{\sqrt[4]{\left(k_{2}^{*} a\right)^{2}-\nu^{2}}} \sin \left[\sqrt{\left(k_{2}^{*} a\right)^{2}-\nu^{2}}-\nu \cos ^{-1}\left(\frac{\nu}{k_{2}^{*} a}\right)+\frac{\pi}{4}\right] \\
& Y_{\nu}\left(k_{2}^{*} a\right) \sim-\sqrt{\frac{2}{\pi}} \frac{1}{\sqrt[4]{\left(k_{2}^{*} a\right)^{2}-\nu^{2}}} \cos \left[\sqrt{\left(k_{2}^{*} a\right)^{2}-\nu^{2}}-\nu \cos ^{-1}\left(\frac{\nu}{k_{2}^{*} a}\right)+\frac{\pi}{4}\right] \\
& J_{\nu}^{\prime}\left(k_{2}^{*} a\right) \sim \sqrt{\frac{2}{\pi}} \frac{\sqrt[4]{\left(k_{2}^{*} a\right)^{2}-\nu^{2}}}{k_{2}^{*} a} \cos \left[\sqrt{\left(k_{2}^{*} a\right)^{2}-\nu^{2}}-\nu \cos ^{-1}\left(\frac{\nu}{k_{2}^{*} a}\right)+\frac{\pi}{4}\right] \\
& Y_{\nu}^{\prime}\left(k_{2}^{*} a\right) \sim \sqrt{\frac{2}{\pi}} \frac{\sqrt[4]{\left(k_{2}^{*} a\right)^{2}-\nu^{2}}}{k_{2}^{*} a} \sin \left[\sqrt{\left(k_{2}^{*} a\right)^{2}-\nu^{2}}-\nu \cos ^{-1}\left(\frac{\nu}{k_{2}^{*} a}\right)+\frac{\pi}{4}\right] \\
& J_{\nu}\left(k_{2}^{*} b\right) \sim \sqrt{\frac{2}{\pi}} \frac{1}{\sqrt[4]{\left(k_{2}^{*} b\right)^{2}-\nu^{2}}} \sin \left[\sqrt{\left(k_{2}^{*} b\right)^{2}-\nu^{2}}-\nu \cos ^{-1}\left(\frac{\nu}{k_{2}^{*} b}\right)+\frac{\pi}{4}\right] \\
& Y_{\nu}\left(k_{2}^{*} b\right) \sim-\sqrt{\frac{2}{\pi}} \frac{1}{\sqrt[4]{\left(k_{2}^{*} b\right)^{2}-\nu^{2}}} \cos \left[\sqrt{\left(k_{2}^{*} b\right)^{2}-\nu^{2}}-\nu \cos ^{-1}\left(\frac{\nu}{k_{2}^{*} b}\right)+\frac{\pi}{4}\right] .
\end{aligned}
$$

Where $J_{\nu}(x)$ and $Y_{\nu}(x)$ denote the Bessel function and the Neumann function, respectively [9]. The prime $\left({ }^{\prime}\right)$ on these functions denotes the derivative with respect to the argument. 